\title{
Frequency comb generation through the locking of domain walls in doubly resonant dispersive optical parametric oscillators
}

\author{
Pedro Parra-Rivas ${ }^{1,2,}{ }^{*}$, Lendert Gelens ${ }^{2}$, Tobias Hansson ${ }^{3}$, Stefan Wabnitz ${ }^{4,5}$, and \\ FRANÇOIS LEO ${ }^{1}$ \\ ${ }^{1}$ OPERA-photonics, Université libre de Bruxelles, 50 Avenue F. D. Roosevelt, CP 194/5, B-1050 Bruxelles, Belgium \\ ${ }^{2}$ Laboratory of Dynamics in Biological Systems, KU Leuven Department of Cellular and Molecular Medicine, University of Leuven, B-3000 Leuven, Belgium \\ ${ }^{3}$ Department of Physics, Chemistry and Biology, Linköping University, SE-581 83 Linköping, Sweden \\ ${ }^{4}$ Dipartimento di Ingegneria dell'Informazione, Elettronica e Telecomunicazioni, Sapienza University of Rome, Via Eudossiana 18, 00184 Rome, Italy \\ ${ }^{5}$ Istituto Nazionale di Ottica del CNR, via Branze 38, 25123 Brescia, Italy \\ ${ }^{*}$ Corresponding author:
}

Compiled December 23, 2018

In this letter we theoretically investigate the formation of localized temporal dissipative structures, and their corresponding frequency combs in doubly resonant dispersive optical parametric oscillators. We derive a nonlocal mean field model, and show that domain wall locking allows for the formation of stable coherent optical frequency combs. (๑) 2018 Optical Society of America

OCIS codes: (190.4410) Nonlinear optics, parametric processes; (190.5530) Pulse propagation and temporal solitons; (140.4780) Optical resonators.

\section{http://dx.doi.org/10.1364/XX.XX.XXXXXX}

The formation of optical frequency combs (OFCs) in high-Q driven microresonators with Kerr type nonlinearity has attracted considerable attention in the past ten years $[1,2]$. Recent works show that dissipative cavities with quadratic nonlinearities may provide an alternative to Kerr cavities for the generation of frequency combs [3-5]. Their main advantage is the possibility of generating combs in previously unattainable spectral regions. For example, optical parametric oscillators (OPOs) may allow for the generation of frequency combs in the mid-infrared, from near-infrared continuous wave sources. It was recently shown that modulation instability (MI) induces pattern and frequency comb formation in denegerate OPOs [6]. While promising, it is still unclear whether solitary waves exist in dispersive OPOs.

In this letter, we propose the locking of domain walls (DWs), also called wave fronts, as an alternative mechanism to MI for the generation of OFCs in temporal degenerate OPOs. A DW consist of a transition connecting two different but coexisting continuous wave $(\mathrm{CW})$ or homogeneous states. DWs can exist separately, or lock forming localized structures (LSs) [7]. The mechanism of DW locking was widely studied in the context of normal dispersion Kerr combs [8-10]. More recently, it was shown that DWs may form between two polarisation states in fiber resonators [11]. Similar structures are well known to appear in spatial optical parametric oscillators [12-14]. DWs stem from the pi phase indeterminacy of the field above threshold. Both solutions may coexist, forming a wall between states of different phases. In this work, we show that similar structures appear in temporal optical parametric oscillators, and that their formation can be easily understood within the framework of a single parametrically forced Ginzburg-Landau equation. We use the system parameters of a recent experiment, demonstrating MIinduced OFCs in singly resonant optical parametric oscillators [6].

To start, let us consider a dispersive cavity with a quadratic medium phase-matched for degenerate OPO, and driven by the field $B_{i n}$ at frequency $2 \omega_{0}$ in a doubly resonant configuration. Such a system can be described by an infinite map for the slowly varying envelopes of the fields $A_{m}(z, t)$ and $B_{m}(z, t)$, of the electric field

$$
E_{m}(z, t)=\operatorname{Re}\left[A_{m}(z, t) e^{i\left(k_{1} z-\omega_{0} t\right)}+B_{m}(z, t) e^{i\left(k_{2} z-2 \omega_{0} t\right)}\right],
$$

centered at frequencies $\omega_{0}$ and $2 \omega_{0}$, respectively. Propagation of these cavity fields over the $m^{\text {th }}$ round trip is governed by the evolution equations:

$$
\begin{array}{r}
\partial_{z} A_{m}=-\left(\frac{\alpha_{c 1}}{2}+i \frac{k_{1}^{\prime \prime}}{2} \partial_{\tau}^{2}\right) A_{m}+i \kappa B_{m} \bar{A}_{m} e^{-i \Delta k z} \\
\partial_{z} B_{m}=-\left(\frac{\alpha_{c 2}}{2}+\Delta k^{\prime} \partial_{\tau}+i \frac{k_{2}^{\prime \prime}}{2} \partial_{\tau}^{2}\right) B_{m}+i \kappa A_{m}^{2} e^{i \Delta k z},
\end{array}
$$

where $\tau=t-z / v_{g}$, and $v_{g}=1 / k_{1}^{\prime}$ is the group velocity of the fundamental field $A_{m}$. Here $z \in\left[0, L_{c}\right]$ with $L_{c}$ the length of the cavity; $\alpha_{c 1,2}$ describe linear propagation losses for the fields $A_{m}$ and $B_{m} ; k_{1,2}^{\prime \prime}=d^{2} k /\left.d \omega^{2}\right|_{\omega_{0}, 2 \omega_{0}}$ are the group velocity dispersion (GVD) coefficients; $\Delta k=2 k(\omega)-k\left(2 \omega_{0}\right)$ is the wave-vector mismatch at the degeneracy point; $\Delta k^{\prime}=d k /\left.d \omega\right|_{2 \omega_{0}}-d k /\left.d \omega\right|_{\omega_{0}}$ is the corresponding group-velocity mismatch, or temporal walk-off; and the nonlinear coupling strength $\kappa \propto \chi^{(2)}$ is normalized such that $\left|A_{m}\right|^{2},\left|B_{m}\right|^{2}$ and $\left|B_{i n}\right|^{2}$ are measured in Watts. Furthermore, the intracavity fields $A_{m+1}(0, \tau)$ and 


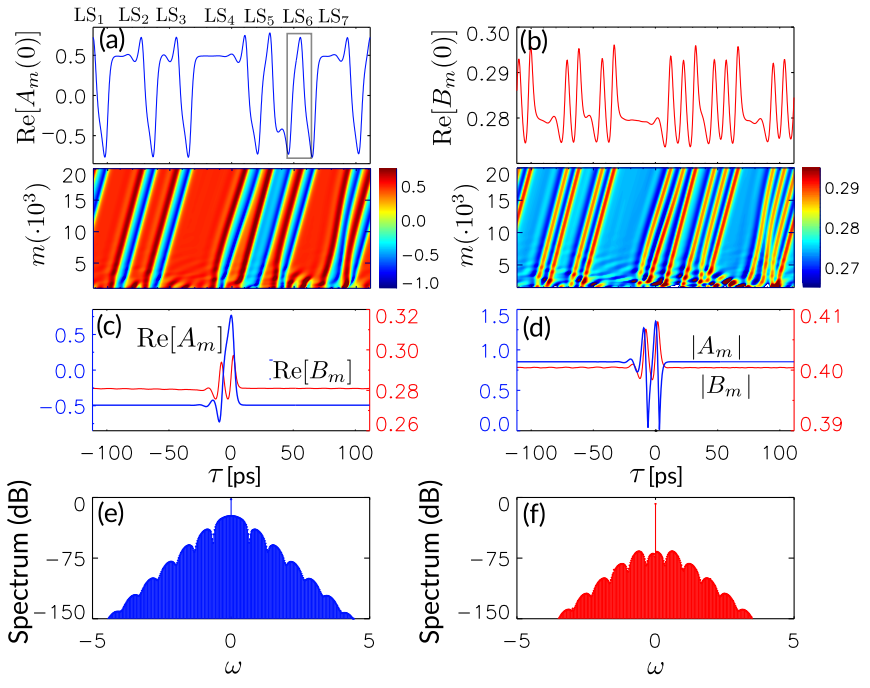

Fig. 1. In (a) and (b) the evolution after each round-trip of initial random noise [bottom], and formation of a stationary dissipative disordered structure for $m=2 \cdot 10^{4}$ [top], are shown plotting $\operatorname{Re}\left[A_{m}(z=0)\right]$ (blue) and $\operatorname{Re}\left[B_{m}(z=0)\right]$ (red) in $\mathrm{W}^{1 / 2}$. (c)-(d) Show the real part and absolute value of a single bump LS, respectively. (e) - (f) Quadratic OFCs associated with $A_{m}(z=0)$ and $B_{m}(z=0)$. Physical parameters used: $\kappa=6.58$ $\mathrm{W}^{-1 / 2} / \mathrm{m}, \alpha_{1,2}=T_{1,2}=0.0196, k_{1}^{\prime \prime} \approx 0.234 \mathrm{ps}^{2} / \mathrm{m}, k_{2}^{\prime \prime} \approx 0.714$ $\mathrm{ps}^{2} / \mathrm{m}, \Delta k^{\prime} \approx 792 \mathrm{ps} / \mathrm{m}, \delta_{1}=-0.0392, B_{\text {in }}=0.395 \mathrm{~W}^{1 / 2}$, $L_{\mathrm{c}}=0.015 \mathrm{~m}$, and $t_{R} \approx 223 \mathrm{ps}$ [6].

$B_{m+1}(0, \tau)$ at the beginning of the $(m+1)^{\text {th }}$ round-trip are related to the fields at the end of the $m^{\text {th }}$ round-trip by

$$
\begin{gathered}
A_{m+1}(0, \tau)=\sqrt{1-T_{1}} A_{m}\left(L_{c}, \tau\right) e^{-i \delta_{1}} \\
B_{m+1}(0, \tau)=\sqrt{1-T_{2}} B_{m}\left(L_{c}, \tau\right) e^{-i \delta_{2}}+\sqrt{T_{2}} B_{i n},
\end{gathered}
$$

where $T_{1}$ and $T_{2}$ are the power transmission coefficients, at $\omega_{0}$ and $2 \omega_{0}$, of the coupler used to inject the CW field $B_{i n}$, and $\delta_{j}=\left(\omega_{j}-\omega_{c}\right) t_{R}$ with $j=1,2$ represents the phase detuning of the intracavity field $A(B)$ for the cavity resonance frequency $\omega_{c}$ closest to $\omega_{1}=\omega_{0}\left(\omega_{2}=2 \omega_{0}\right)$, over one round-trip time $t_{R}$. In the following we consider the case of a perfect phase matching $\Delta k=0, \delta_{2}=2 \delta_{1}$ [5]. We use the map (2-3) to numerically explore the natural dynamics of the system. Figs. 1(a) and (b) show the evolution of an initial noisy background after each round-trip (bottom), and the formation of a dissipative structure after a sufficient number of round-trips $\left(m=2 \cdot 10^{4}\right)$ (top) [15]. In both cases the real parts of the fields $A$ and $B$ are plotted in blue and red, respectively. As one can appreciate, both fields are phase-matched and drift at the same constant velocity. Looking to the $A$ field [see Fig. 1(a)], we can identify a sequence of DWs connecting two different continuous wave states, forming a disordered stationary state. This particular solution consists of seven LSs (see $\mathrm{LS}_{1}-\mathrm{LS}_{7}$ ) of different widths and separations between them. At the location of each of the DWs in the $A$ field, one finds pulses in the $B$ field [see Figure 1(b)]. Furthermore, one can use each such LS as a new initial condition for the map, and find that each LS is also a localized steady state solution of the system [see for instance $\mathrm{LS}_{6}$ in Figure 1(c)]. Moreover, when starting with a noisy background and the same parameter set, we find that different realizations of the noise can lead to many different sequences of such pulses, revealing multistability of LSs in the system. Panel (d) shows the absolute values of $A$ and $B$, and the spectra associated with both fields are plotted in panels (e) and (f). As far as we know, the existence of this type of structures has not yet been reported in the context of quadratic dispersive cavities. Therefore, it is important to elucidate their formation and properties. For doing so, we first derive a single time-domain equation that allows for the description of stationary states associated with LSs, and their spectral properties.

Assuming that the resonator exhibits high finesse, that both fields do not vary significantly over a single round-trip (i.e., the combined effects of nonlinearity and dispersion are weak), and following Refs. $[5,16,17]$ one can reduce Eqs. (2) and (3), to two coupled mean-field equations:

$$
\begin{gathered}
\partial_{t} A=-\left(1+i \theta_{1}\right) A-i \beta_{1} \partial_{\tau^{\prime}}^{2} A+i \sigma B \bar{A} \\
\partial_{t} B=-\left(\alpha+i \theta_{2}\right) B-\left(d \partial_{\tau^{\prime}}+i \beta_{2} \partial_{\tau^{\prime}}^{2}\right) B+i \bar{\sigma} A^{2}+S,
\end{gathered}
$$

where $A(t, \tau)$ and $B(t, \tau)$ are the normalized cavity field envelopes at $z=0$ defined as $A\left(t=m t_{R}, \tau\right)=\kappa L_{c} A_{m}(z=$ $0, \tau) / \alpha_{1}$ and $B\left(t=m t_{R}, \tau\right)=\kappa L_{c} B_{m}(z=0, \tau) / \alpha_{1}$, and the index $m$ has been replaced by the slow-time variable $t=m t_{R}$. Here the normalized variables and parameters are $\alpha=\alpha_{2} / \alpha_{1}$, where $\alpha_{1,2}=\left(T_{1,2}+\alpha_{c 1,2} L_{c}\right) / 2, \theta_{1,2}=\delta_{1,2} / \alpha_{1}$, $d=\Delta k^{\prime} \sqrt{2 L_{c} / \alpha_{1}\left|k_{1}^{\prime \prime}\right|}, S=B_{\text {in }} \sqrt{T_{2}} \kappa L_{c} / \alpha_{1}^{2}, \beta_{1}=\operatorname{sign}\left(k_{1}^{\prime \prime}\right)$, $\beta_{2}=k_{2}^{\prime \prime} /\left|k_{1}^{\prime \prime}\right|, \sigma=\operatorname{sinc}\left(\Delta k L_{c}\right) e^{-i \Delta k L_{c}}$ and $\tau^{\prime}=\tau \sqrt{2 \alpha_{1} /\left|k_{1}^{\prime \prime}\right| L_{c}}$. The mean-field model (4) can be used to study OFC generation and dynamics in OPOs in detail. Indeed, Eqs. 4 are more suitable than Eqs. (2-3) to perform analytical studies, and permit to apply numerical continuation algorithms. By assuming that $B$ evolves slowly, such that $\partial_{t} B \approx 0$, we can further simplify Eqs. 4 to a single mean-field model, as already done in Ref. [17] for cavity enhanced second-harmonic generation. To do so, from Eq. (4b) one can obtain an expression of $B$ as a function of $A^{2}$ and $S$, that once inserted in Eq. (4a) gives:

$$
\partial_{t} \mathrm{~A}=-\left(1+i \theta_{1}\right) \mathrm{A}-i \beta_{1} \partial_{\tau^{\prime}}^{2} \mathrm{~A}-\overline{\mathrm{A}} \mathrm{A}^{2} \otimes \mathrm{J}+\rho \overline{\mathrm{A}} .
$$

with $\otimes$ denoting the convolution with the nonlocal kernel

$$
\mathrm{J}\left(\tau^{\prime}\right)=\frac{1+\Theta_{2}^{2}}{2 \pi} \int_{-\infty}^{\infty} \frac{e^{-i \Omega \tau^{\prime}} d \Omega}{1+i\left(\Theta_{2}-\gamma \Omega+\eta \Omega^{2}\right)},
$$

where $\Theta_{2}=\theta_{2} / \alpha, \gamma=d / \alpha, \eta=\beta_{2} / \alpha$. The normalized field reads $\mathrm{A}=\sigma A e^{-i \psi} / \sqrt{\alpha\left(1+\Theta_{2}^{2}\right)}$, with $\psi=\pi / 4+$ $\operatorname{atan}\left(-\Theta_{2}\right) / 2$, and the normalized driving field amplitude is $\rho=\sigma S /\left(\alpha \sqrt{1+\Theta_{2}^{2}}\right)$. With this approximation, the $B$ field is dynamically slaved to the $A$ field, and it is explicitly given by $B=-(\mathrm{A} \otimes \mathrm{J}+\rho) e^{i \operatorname{atan}\left(\Theta_{2}\right)} / \sigma$. Without loss of generality we will consider $\beta_{1}=1$, and $\alpha=1$, such that $\Theta_{2}=2 \theta_{1}$.

We have used a variety of steady state solutions obtained with the infinite map (2)-(3), such as those in Fig. 1, as initial condition in the two models (4) and (5). We found these initial temporal profiles to quickly converge, and the converged solutions were almost identical in all models. This confirms the validity of both mean field models (4) and (5). Therefore, for studying the type of structures considered here, one can indistinctly use any of these models in the analysis. In what follows, we will focus our study on the simplest model, provided by Eq. (5).

Based on our observations in Fig. 1, we anticipate that the LSs in panels (a)-(b) [top] are composed of two DWs connecting different $\mathrm{CW}$ solutions that coexist for the same values of 

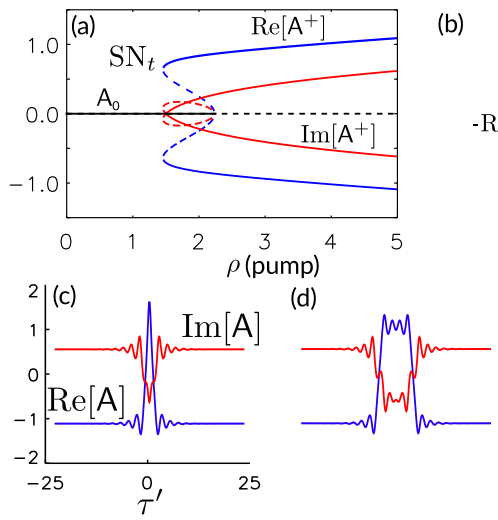

(b)

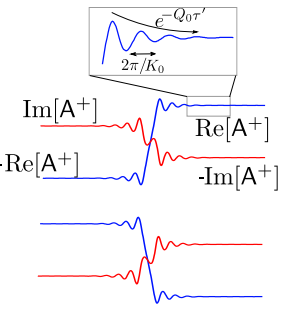

(e)
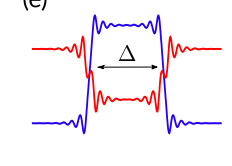

Fig. 2. In (a) we show the HSS solution of Eq. (5) in the subcritical regime for $\theta_{1}=-2$. Panel (b) shows an example of two DW solutions of Eq. (8) with different polarities for $\left(\theta_{1}, \rho\right)=(-2,4)$. For the same parameters, panels (c)-(e) show different LSs formed through the locking of the DWs at different separations. Here $(\gamma, \eta)=(0,0)$.

parameters. These CWs correspond to the homogeneous steady state (HSS) solutions of Eq. (5), namely $A_{0}=0$, and the two branches of solutions $A=A^{ \pm}$satisfying

$$
\left|A^{ \pm}\right|^{2}=\frac{\left(\theta_{1} \Theta_{2}-1\right) \pm \sqrt{\left(1+\Theta_{2}^{2}\right) \rho^{2}-\left(\Theta_{2}+\theta_{1}\right)^{2}}}{1+\Theta_{2}^{2}},
$$

with $\mathrm{A}^{ \pm}=\left|\mathrm{A}^{ \pm}\right| e^{i \phi}$, and $\phi=\arcsin \left(\sqrt{\left(\rho-1-\left|\mathrm{A}^{ \pm}\right|^{2}\right) / 2 \rho}\right)$. For large enough detuning, $\theta_{1}>1 / \Theta_{2}$, only the $A^{+}$branch exists, and bifurcates supercritically from a pitchfork bifurcation at a pump strength $\rho_{a}=\sqrt{1+\theta_{1}^{2}}$. However, if $\theta_{1}<1 / \Theta_{2}, \mathrm{~A}^{-}$ emerges subcritically (and therefore unstably), and stabilizes at a saddle-node $\mathrm{SN}_{t}$, at $\rho_{t}=\left(\Theta_{2}+\theta_{1}\right) / \sqrt{1+\Theta_{2}^{2}}$, where it merges with $\mathrm{A}^{+}$. Without loss of generality we will focus on the subcritical regime, whose bifurcation diagram is shown in Fig. 2(a).

When coupling between points of the fast time variable (i.e. $\tau^{\prime}$ ) is introduced in the system through the dispersion $\partial_{\tau^{\prime}}^{2} \mathrm{~A}$ and/or the nonlocal nonlinearity $A^{2} \otimes \mathrm{J}$, connections between the HSSs can arise. Furthermore, as we discuss later, LSs can form through the locking of these DWs. To illustrate this mechanism, let us first consider the presence of dispersion only. A natural way for doing so is to neglect walk-off and the GVD of $B$, so that $\gamma=0$, and $\eta=0$. In this case, the convolution $A^{2} \otimes J$ reduces to $\left(1-i \Theta_{2}\right) \mathrm{A}^{2}$ and Eq. (5) becomes

$$
\partial_{t} \mathrm{~A}=-\left(1+i \theta_{1}\right) \mathrm{A}-i \beta_{1} \partial_{\tau^{\prime}}^{2} \mathrm{~A}-\left(1-i \Theta_{2}\right)|\mathrm{A}|^{2} \mathrm{~A}+\rho \overline{\mathrm{A}},
$$

which is a simpler version of the well known parametrically forced Ginzburg-Landau equation with 2:1 resonance [18]. Notice that the HSS solutions of this equation are also given by (7). In diffractive systems that are not bounded periodically, DWs connecting the equivalent states $-\mathrm{A}^{+}$and $\mathrm{A}^{+}$can exist in both supercritical and subcritical regimes [12]. Fig. 2(b) shows two examples of a DW in the subcritical regime for $\left(\theta_{1}, \rho\right)=(-2,4)$, one showing an upwards connection of $-\mathrm{A}^{+}$ to $A^{+}$(top), and the other showing a downwards connection from $\mathrm{A}^{+}$to $-\mathrm{A}^{+}$(bottom). We refer to these two types of
DWs as having an opposite polarity. Furthermore, they are invariant under the simultaneous transformations $\tau^{\prime} \mapsto-\tau^{\prime}$, and $A \mapsto-A$, and they are stationary, i.e. they are Ising fronts [19]. Here we restrict our attention to Ising type of DWs, and do not study the case when these DWs undergo a drift bifurcation, where two counter-propagating asymmetric (Bloch) fronts appear [19] and that lead to a more complex dynamics [20].

In order for DWs of opposite polarity to form stable connections, it is of critical importance to consider the way that the wave fronts approach the HSS asymptotically. In the linear regime, this approach can be described by $\mathrm{A}\left(\tau^{\prime}\right)=$ $\mathrm{A}^{+}+a e^{\lambda \tau^{\prime}}+$ c.c., where $|a| \ll 1$ and $\lambda=Q+i K$, with $Q$ and $K$ real numbers. These eigenvalues $\lambda$ depend on the control parameters of the system, and can be obtained by studying the system of linearized ordinary differential equations for the perturbations, derived through direct substitution of the ansatz into Eq. (5) [18, 21]. In the linear regime (i.e. far from the DW core), the overall shape of the DW oscillatory tail that approaches the HSS is determined by the leading eigenvalue $\lambda_{0}=Q_{0}+i K_{0}$, which is the eigenvalue with the real part closest to zero, as all the other directions are damped faster. An example of an oscillatory tail $\left(K_{0} \neq 0\right)$ can be seen in detail in the close-up view of Fig. 2(b). If $\lambda_{0}$ is purely real, the front approaches the HSS monotonically.

In a periodic system like ours, single isolated DWs do not exist: to satisfy the boundary conditions they must necessarily always come in pairs. Two DWs of opposite polarity exhibit an interaction force whose strength decays exponentially with their temporal separation, as described by $\partial_{t} \Delta \sim e^{-Q_{0} \Delta} \cos \left(K_{0} \Delta\right)$ [7], where $\Delta$ represents the separation between both DWs [see Fig. 2(e)]. The stationary solutions of this equation, $\Delta_{n} \equiv$ $2 \pi n / K_{0}$ (with $n \in \mathbb{N}$ ), correspond to the locations at which two DWs can lock to each other, and form a LS of width $\Delta \approx \Delta_{n}$. Thus, for a given set of parameters, a multiple LSs of different widths can coexist, as illustrated in Fig. 2(c)-(e). These LSs only form when the DWs approach the HSS in an oscillatory way $\left(K_{0} \neq 0\right)$. In contrast, when the tails are monotonic $\left(K_{0}=0\right)$, two DWs attract each other, and move towards each other until they annihilate one another in a process called coarsening [22]. When either the GVD of the $B$ field, $\eta$, and/or the walk-off, $\gamma$, are present, nonlocal coupling must be taken into account, and one has to consider the more general Eq. (5). In this model, DW solutions still exist, although their shape and symmetry properties are modified, since nonlocality can significantly alter the eigenvalues $\lambda[23,24]$. Nevertheless, the mechanism of DW locking remains the same for solutions of Eq. (5), and similar LSs can be found. To illustrate this, we explore the influence of the walk-off $\gamma$ on the LSs that we found using the local model [see Fig. 2] in the absence of GVD $\eta$ of the $B$ field. The walkoff breaks the left/right symmetry $\left(\tau \mapsto-\tau^{\prime}\right)$, and LSs now drift at a constant velocity $c$ proportional to $\gamma$. Considering the change of coordinates to the moving reference frame (i.e. $\tau^{\prime} \mapsto \tau^{\prime}-c t$ ) and using a numerical continuation algorithm, based on a Newton-Raphson solver, the LSs and their velocity $c$ can be continuously tracked in the parameter $\gamma$. In this way, the bifurcation diagram in Fig. 3 was constructed, where we tracked two LS of different widths [i.e. the solutions shown in Fig. 2(c) and Fig. 2(d)]. The diagram at the top of Fig. 3 shows how the width (blue) and the velocity $c$ (red) of these LS solutions change with increasing walk-off $\gamma$. The solid lines corresponds to the continuation of a narrow LS [Fig. 2(c)], while the dashed 

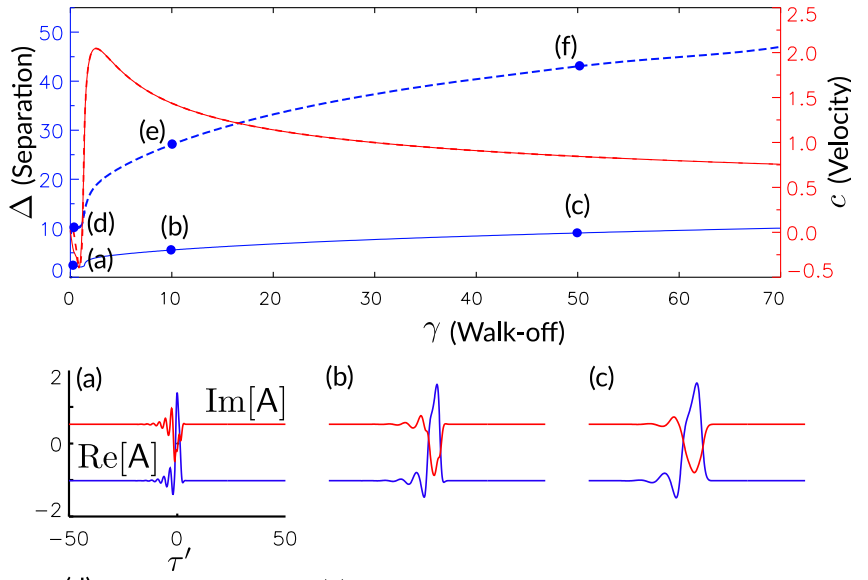

(d)

(e)
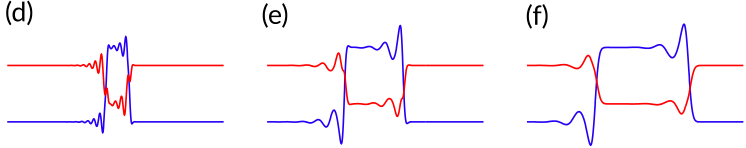

Fig. 3. Bifurcation diagram showing the influence of walk-off $\gamma$ on a single bump LS in the absence of $\eta$ (the GVD of $B$ ). Here the width $\Delta$ and speed $c$ of the LS are shown as a function of $\gamma$. The labels (a)-(d) correspond to the LSs profiles shown in the subpanels. Here $\left(\theta_{1}, \rho\right)=(-2,4)$.

lines show the changes of a wider LS [Fig. 2(d)]. Changes in the profiles of the LSs are illustrated in the subpanels (a)-(f). In both cases, the width of LSs increases monotonically with $\gamma$, due to an increase in the wavelength of the oscillatory tails of the DWs. The velocities of both LSs are approximately equal, indicating that the velocity depends mostly on the strength of the walk-off, and not on the shape and width of the structures. The LS velocity, initially negative, becomes positive at $\gamma \approx 2$, then sharply increases until reaching a peak value $c \approx 2$, after which it decreases monotonically and saturates for large values of the walk-off $\gamma$. For large walk-off, the LSs of Fig. 3 closely resemble the structures that we found by simulating the full map, as shown in Fig. 1. The fact that this type of LSs exists for very large values of walk-off, in contrast to other type of LSs, can have a big practical advantage since no dispersion engineering is needed other than for the phase-matching. By incorporating both walk-off $\gamma$ and GVD $\eta$, we can then retrieve exactly the LSs in Fig. 1. In the absence of $\gamma$, the presence of $\eta$ induces modifications in the tails of the LSs. For the parameter values considered in this work, however, $\gamma$ dominates and the effect of $\eta$ is almost negligible. The influence of the different control parameters of the system on the bifurcation structure of the LSs can be quite complex and their study is beyond the scope of this paper.

In summary, we have studied the formation of temporally LSs in doubly resonant dispersive optical parametric oscillators. Using an infinite map for the slowly varying envelopes of the OPO fields, we showed that a random sequence of LSs of different widths is formed naturally. We then derived a nonlocal mean-field model that describes the dynamics of these OPO cavities, in which we confirmed the existence of localized dissipative structures. Moreover, we demonstrated that these type of structures also exist in the absence of walkoff and GVD of the pump field $B$. They are formed through the locking of DWs connecting two equivalent homogeneous states, which could be explained more easily in the local case.
Afterwards, using continuation techniques, we characterized how these LSs are altered by walk-off and GVD of the pump field $B$. Remarkably, the formation of this type of LSs does not depend on modulational instabilities. In the frequency domain, LSs correspond to coherent frequency combs formed around both $\omega_{0}$ and $2 \omega_{0}$. We expect that these structures may play a significant role for future integrated ultra-broadband frequency comb generation.

Acknowledgment. We acknowledge the support from internal Funds from KU Leuven and the FNRS (PPR); the Ministero dell' Istruzione, dell' Università e della Ricerca (MIUR), [Grant 2015KEZNYM], (SW); funding from the Swedish Research Council, [Grant No. 2017-05309], (TH); funding from the European Research Council (ERC) under the European Union's Horizon 2020 research and innovation programme [Grant agreement No. 757800], (FL).

\section{REFERENCES}

1. P. Del'Haye, A. Schliesser, O. Arcizet, T. Wilken, R. Holzwarth, and T. J. Kippenberg, Nature 450, 1214-1217 (2007).

2. A. Pasquazi, M. Peccianti, L. Razzari, D. J. Moss, S. Coen, M. Erkintalo, Y. K. Chembo, T. Hansson, S. Wabnitz, P. Del'Haye, X. Xue, A. M. Weiner, and R. Morandotti, Phys. Reports 729, 1-81 (2018).

3. I. Ricciardi, S. Mosca, M. Parisi, P. Maddaloni, L. Santamaria, P. De Natale, and M. De Rosa, Phys. Rev. A 91, 063839 (2015).

4. F. Leo, T. Hansson, I. Ricciardi, M. De Rosa, S. Coen, S. Wabnitz, and M. Erkintalo, Phys. Rev. Lett. 116, 033901 (2016).

5. F. Leo, T. Hansson, I. Ricciardi, M. De Rosa, S. Coen, S. Wabnitz, and M. Erkintalo, Phys. Rev. A 93, 043831 (2016).

6. S. Mosca, M. Parisi, I. Ricciardi, F. Leo, T. Hansson, M. Erkintalo, P. Maddaloni, P. De Natale, S. Wabnitz, and M. De Rosa, Phys. Rev. Lett. 121, 093903 (2018).

7. P. Coullet, C. Elphick, and D. Repaux, Phys. Rev. Lett. 58, 431 (1987).

8. V. E. Lobanov, G. Lihachev, T. J. Kippenberg, and M. L. Gorodetsky, Opt. Express 23, 7713 (2015).

9. P. Parra-Rivas, D. Gomila, E. Knobloch, S. Coen, and L. Gelens, Opt. Lett. 41, 2402 (2016)

10. P. Parra-Rivas, E. Knobloch, D. Gomila, and L. Gelens, Phys. Rev. A 93, 063839 (2016).

11. S. Coen, B. Garbin, J. Fatome, Y. Wang, F. Leo, G.-L. Oppo, S. G. Murdoch, and M. Erkintalo,CLEO Pacific Rim Conference 2018, paper Th4B.1.

12. S. Trillo, M. Haelterman, and, A. Sheppard, Op. Lett. 22, 13 (1997).

13. V. B. Taranenko, K. Staliunas, and C. O. Weiss, Phys. Rev. Lett. 81, 2236 (1998).

14. Gian-Luca Oppo, Andrew J Scroggie and William J Firth, J. Opt. B: Quantum Semiclass. Opt. 1 133-138 (1999).

15. The numerical integration of the different models has been performed using a pseudo spectral method in a one-dimensional grid of $N=1024$ points.

16. M. Haelterman, S. Trillo, and S. Wabnitz, Opt. Comm. 91, 401 (1992).

17. T. Hansson, F. Leo, M. Erkintalo, S. Coen, I. Ricciardi, M. De Rosa, and S. Wabnitz, Phys. Rev. A 95, 013805 (2017).

18. J. Burke, A. Yochelis, and E. Knobloch, SIAM J. Appl. Dyn. Syst. 7, 651-711 (2008).

19. P. Coullet, J. Lega, B. Houchmanzadeh, and J. Lajzerowicz, Phys. Rev. Lett. 65, 1352 (1990).

20. D. Gomila, P. Colet, and D. Walgraef, Phys. Rev. Lett., 114, 084101 (2015).

21. G. L. Oppo, A. J. Scroggie, and W. J. Firth, Phys. Rev. E 63, 066209 (2001).

22. J. W. Cahn and J. E. Hilliard, J. Chem. Phys. 28, 258 (1958).

23. M. G. Clerc, D. Escaff, and V. M. Kenkre, Phys. Rev. E. 72, 056217 (2005).

24. L. Gelens, D. Gomila, G. van der Sande, M. A. Matias, and P. Colet, Phys. Rev. Lett. 104, 154101 (2010). 


\section{REFERENCES}

1. P. Del'Haye, A. Schliesser, O. Arcizet, T. Wilken, R. Holzwarth, and T. J. Kippenberg, "Optical frequency comb generation from a monolithic microresonator," Nature 450, 1214-1217 (2007).

2. A. Pasquazi, M. Peccianti, L. Razzari, D. J. Moss, S. Coen, M. Erkintalo, Y. K. Chembo, T. Hansson, S. Wabnitz, P. Del'Haye, X. Xue, A. M. Weiner, and R. Morandotti, "Micro-combs: A novel generation of optical sources," Phys. Reports 729, 1-81 (2018).

3. I. Ricciardi, S. Mosca, M. Parisi, P. Maddaloni, L. Santamaria, P. De Natale, and M. De Rosa, "Frequency comb generation in quadratic nonlinear media," Phys. Rev. A 91, 063839 (2015).

4. F. Leo, T. Hansson, I. Ricciardi, M. De Rosa, S. Coen, S. Wabnitz, and M. Erkintalo, "Walk-Off-Induced Modulation Instability, Temporal Pattern Formation, and Frequency Comb Generation in CavityEnhanced Second-Harmonic Generation," Phys. Rev. Lett. 116, 033901 (2016).

5. F. Leo, T. Hansson, I. Ricciardi, M. De Rosa, S. Coen, S. Wabnitz, and M. Erkintalo, "Frequency-comb formation in doubly resonant secondharmonic generation," Phys. Rev. A 93, 043831 (2016).

6. S. Mosca, M. Parisi, I. Ricciardi, F. Leo, T. Hansson, M. Erkintalo, P. Maddaloni, P. De Natale, S. Wabnitz, and M. De Rosa, "Modulation Instability Induced Frequency Comb Generation in a Continuously Pumped Optical Parametric Oscillator", Phys. Rev. Lett. 121, 093903 (2018).

7. P. Coullet, C. Elphick, and D. Repaux,"Nature of Spatial Chaos", Phys. Rev. Lett. 58, 431 (1987).

8. V. E. Lobanov, G. Lihachev, T. J. Kippenberg, and M. L. Gorodetsky, "Frequency combs and platicons in optical microresonators with normal GVD", Opt. Express 23, 7713 (2015).

9. P. Parra-Rivas, D. Gomila, E. Knobloch, S. Coen, and L. Gelens, "Origin and stability of dark pulse Kerr combs in normal dispersion resonators", Opt. Lett. 41, 2402 (2016).

10. P. Parra-Rivas, E. Knobloch, D. Gomila, and L. Gelens, "Dark solitons in the Lugiato-Lefever equation with normal dispersion", Phys. Rev. A 93, 063839 (2016).

11. S. Coen, B. Garbin, J. Fatome, Y. Wang, F. Leo, G.-L. Oppo, S. G. Murdoch, and M. Erkintalo "Dissipative polarization domain walls as persisting topological defects",CLEO Pacific Rim Conference 2018, paper Th4B.1.

12. S. Trillo, M. Haelterman, and, A. Sheppard, "Stable topological spatial solitons in optical parametric oscillators", Op. Lett. 22, 13 (1997).

13. V. B. Taranenko, K. Staliunas, and C. O. Weiss,"Pattern Formation and Localized Structures in Degenerate Optical Parametric Mixing" Phys. Rev. Lett. 81, 2236 (1998).

14. Gian-Luca Oppo, Andrew J Scroggie and William J Firth, "From domain walls to localized structures in degenerate optical parametric oscillators", J. Opt. B: Quantum Semiclass. Opt. 1, 133-138 (1999).

15. The numerical integration of the different models has been performed using a pseudo spectral method in a one-dimensional grid of $N=1024$ points.

16. M. Haelterman, S. Trillo, and S. Wabnitz, "Dissipative modulation instability in a nonlinear dispersive ring cavity", Opt. Comm. 91, 401 (1992).

17. T. Hansson, F. Leo, M. Erkintalo, S. Coen, I. Ricciardi, M. De Rosa, and S. Wabnitz, "Singly resonant second-harmonic-generation frequency combs," Phys. Rev. A 95, 013805 (2017).

18. J. Burke, A. Yochelis, and E. Knobloch, "Classification of spatially localized oscillations in periodically forced dissipative systems", SIAM J. Appl. Dyn. Syst. 7, 651-711 (2008).

19. P. Coullet, J. Lega, B. Houchmanzadeh, and J. Lajzerowicz,'Breaking chirality in nonequilibrium systems' Phys. Rev. Lett. 65, 1352 (1990).

20. D. Gomila, P. Colet, and D. Walgraef,"Theory for the Spatiotemporal Dynamics of Domain Walls close to a Nonequilibrium Ising-Bloch Transition", Phys. Rev. Lett., 114, 084101 (2015).

21. G. L. Oppo, A. J. Scroggie, and W. J. Firth,"Characterization, dynamics and stabilization of diffractive domain walls and dark ring cavity solitons in parametric oscillators", Phys. Rev. E 63, 066209 (2001).

22. J. W. Cahn and J. E. Hilliard, 'Free Energy of a Nonuniform System. I.
Interfacial Free Energy ', J. Chem. Phys. 28, 258 (1958)

23. M. G. Clerc, D. Escaff, and V. M. Kenkre, "Patterns and localized structures in population dynamics", Phys. Rev. E. 72, 056217 (2005).

24. L. Gelens, D. Gomila, G. van der Sande, M. A. Matias, and P. Colet, "Nonlocality-Induced Front-Interaction Enhancement", Phys. Rev. Lett. 104, 154101 (2010). 